\title{
Association of Copper Status with Lipid Profile and Functional Status in Patients with Amyotrophic Lateral Sclerosis
}

\author{
Acsa Nara A. B. Barros, ${ }^{1}$ Mário Emílio T. Dourado Jr., ${ }^{2}$ Lucia de Fatima C. Pedrosa, \\ and Lucia Leite-Lais $\mathbb{D}^{1}$ \\ ${ }^{1}$ Department of Nutrition, Federal University of Rio Grande do Norte, Natal, RN, Brazil \\ ${ }^{2}$ Department of Medicine, Federal University of Rio Grande do Norte, Natal, RN, Brazil \\ Correspondence should be addressed to Lucia Leite-Lais; ludl10@hotmail.com
}

Received 6 March 2018; Accepted 14 June 2018; Published 19 July 2018

Academic Editor: Michael B. Zemel

Copyright (C) 2018 Acsa Nara A. B. Barros et al. This is an open access article distributed under the Creative Commons Attribution License, which permits unrestricted use, distribution, and reproduction in any medium, provided the original work is properly cited.

\begin{abstract}
Oxidative stress is one of the main mechanisms associated with the pathogenesis of amyotrophic lateral sclerosis (ALS). Copper can affect cellular oxidation and lipid metabolism. The aim of this study was to evaluate the association of copper status with lipid profile and functional status in patients with ALS. A cross-sectional study was carried out including 27 patients with ALS (case group) and 26 healthy individuals (control group). Copper status was evaluated by habitual dietary copper intake, plasma copper, and serum ceruloplasmin concentrations. The lipid profile included analysis of serum total cholesterol (TC), LDL-cholesterol (LDL-c), HDL-cholesterol (HDL-c), and triglycerides (TGL). The functional status of patients with ALS was assessed by the ALS Functional Rating Scale-Revised (ALSFRS-R). In the case group, plasma copper was lower compared with the control group (133.9 versus $164.1 \mu \mathrm{g} / \mathrm{dL}, p=0.0001)$ and was positively correlated with HDL-c $\left(r_{\mathrm{s}}=0.398, p=0.044\right)$. In the control group, plasma copper was positively correlated with serum ceruloplasmin $\left(r_{\mathrm{s}}=0.646, p<0.001\right)$, TC $\left(r_{\mathrm{s}}=0.446, p=0.025\right), \mathrm{LDL}-\mathrm{c}\left(r_{\mathrm{s}}=0.445\right.$, $p=0.029)$, and HDL-c $\left(r_{\mathrm{s}}=0.479, p=0.015\right)$, and serum ceruloplasmin was positively correlated only with LDL-c $\left(r_{\mathrm{s}}=0.407\right.$, $p=0.043)$. In the case group, dietary copper intake $(B=-0.373, p<0.001)$, plasma copper $(B=-0.005, p=0.033)$, and TC $(B=-0.312, p=0.001)$ were inversely associated with the functional status of patients with ALS. In contrast, serum ceruloplasmin $(B=0.016, p=0.044)$, LDL-c $(B=0.314, p=0.001)$, HDL-c $(B=0.308, p=0.001)$, and TGL $(B=0.062 ; p=0.001)$ were positively associated with their functional status. In conclusion, this study suggests a disturbance of copper status and its connection with the lipid profile in patients with ALS. Furthermore, copper status and lipid profile may influence the functional status of patients with ALS, standing out as potential biomarkers of disease severity.
\end{abstract}

\section{Introduction}

Amyotrophic lateral sclerosis (ALS) is a progressive and fatal disease characterized by degeneration of motor neurons in the brain and spinal cord, leading to skeletal muscle atrophy, paralysis, and death. The incidence of ALS is about $1 / 100,000$, with a survival time since onset ranging from 24 to 48 months and an estimated mortality of 30,000 patients a year worldwide [1]. The etiology of ALS remains unknown. However, several mechanisms have been implicated in ALS pathophysiology and progression, such as oxidative stress, glutamate excitotoxicity, mitochondrial dysfunction, neuroinflammation, and protein aggregation [2].
Abnormal metal homeostasis may have an important role in the onset and progression of neurodegenerative disorders [3]. In this sense, copper has been extensively studied and plays an important role in the central nervous system (CNS). This micronutrient is essential for angiogenesis, myelination, neurotransmission, cellular respiration, and antioxidant defense. Thus, disturbances in copper homeostasis generate functional impairments in the CNS, including neurodegeneration [4], one of the characteristics found in ALS. In addition, both copper excess and deficiency contribute to oxidative stress, a key component of ALS progression [5]. Copper excess catalyzes biochemical reactions that produce reactive oxygen species. Copper 
deficiency, in turn, leads to poor activity of copperdependent antioxidant enzymes, such as superoxide dismutase (SOD), glutathione peroxidase (GSH-Px), and metallothionein [6]. Copper-mediated oxidative stress is associated with changes in genes expression, lipoprotein structure, membrane receptors, and consequently lipid profile [7].

Some studies have demonstrated that higher serum lipid concentrations are associated with slower progression, better prognosis, and prolonged survival in ALS patients [8-11]. The link between copper status and lipid metabolism has been reviewed $[7,12,13]$. Clinical studies have found different associations between serum copper and lipids, depending on the population studied and the presence of comorbidities [14-19]. Thus, both copper excess and deficiency not only contribute to oxidative stress but also alter serum lipid concentrations [7].

Copper status and lipid profile have been studied in ALS patients separately; however, these two subjects have not been investigated together in these patients. Considering the scarcity of studies on this subject, the present study aimed at evaluating the association of copper status with lipid profile and functional status in patients with ALS.

\section{Materials and Methods}

2.1. Participants and Study Design. This cross-sectional study was reviewed and approved by the Ethics Committee of the Onofre Lopes University Hospital in Natal, Brazil (CAAE 40467214.0.0000.5292). All subjects provided written, informed consent before enrollment.

The case group consisted of patients with ALS treated at the multidisciplinary outpatient facility at the Onofre Lopes University Hospital in Natal, Brazil, between March 2016 and December 2016. Inclusion criteria were patients of both sexes with probable or definite ALS diagnosis and under the care of a literate family member or caregiver. Exclusion criteria were patients with suspected or possible ALS diagnosis, taking micronutrient supplements, undergoing estrogen treatment, and with another neurological disease, inflammatory bowel disease, biliary diseases, thyroid dysfunction, diabetes mellitus, and renal and hepatic diseases. These exclusion criteria were adopted due to their possible influence on copper status.

For comparison purposes, healthy adults and elderly individuals were recruited as the control group. The recruitment of the control group occurred through social media invitations sent to the local community. A matching based on age and sex was adopted to improve the quality of the data. The same exclusion criteria were adopted for the control group.

2.2. Clinical Characterization. Patients with ALS were characterized by the onset site of symptoms (bulbar or spinal), time of symptoms (in months), feeding route (oral and/or enteral), and functional status using the ALS Functional Rating Scale-Revised (ALSFRS-R) [20] and validated to the Portuguese language [21]. This instrument evaluates the progression of patients' disability and the severity of the disease through a 12-item questionnaire, covering aspects related to dysphagia, daily life activities, and respiratory function. The overall ALSFRS-R score ranged from 0 to 48, where 0 represents the worst stage of disability and 48 represents the normal functional status.

2.3. Anthropometric Assessment. The anthropometric assessment was performed utilizing the body mass index (BMI) [22]. The body weight was measured in a calibrated digital Knwaagen ${ }^{\circledR}$ scale with maximum capacity of $500 \mathrm{~kg}$. The height was measured using a Medjet ${ }^{\circledR}$ stadiometer. For wheelchair patients, the height was estimated according to Chumlea et al. $[23,24]$.

\subsection{Copper Status and Lipid Profile Evaluation}

2.4.1. Dietary Copper Intake. Habitual dietary copper intake was investigated using two nonconsecutive 24 -hour recalls for each participant, obtained from weekdays, 30 to 45 days apart [25]. Food intake data were calculated using Virtual Nutri Plus ${ }^{\circledR} 2.0$ software. Food items or preparations that were not found in the software's databank were added based on the nutrition labels of industrialized products. Habitual dietary copper intake was estimated after adjustments of interpersonal variability, according to Nusser et al. [26]. Next, copper intake was adjusted for energy, applying the residual method described by Willett et al. [27]. The estimated average requirement (EAR) for copper $(0.7 \mathrm{mg} /$ day $)$ [28] was used as a parameter to assess copper intake.

\subsubsection{Plasma Copper, Serum Ceruloplasmin, and Lipid} Profile. After an overnight fast, blood samples were collected from participants in specific tubes to perform all hematological analysis. For plasma copper evaluation, blood samples were collected in metal-free tubes with $100 \mu \mathrm{L}$ of $30 \%$ sodium citrate anticoagulant. For serum ceruloplasmin evaluation, blood samples were collected in tubes with gel clot activator (BD Vacutainer ${ }^{\circledR}$ SST $^{\mathrm{TM}}$ II Advance, BD, UK). For lipid profile evaluation, blood samples were collected in tubes with gel clot activator (VacuTube, Biocon, BR).

Plasma copper concentrations were determined by atomic absorption spectrophotometry (SpectrAA200, Varian, Victoria, Australia). The samples were centrifuged at $3000 \mathrm{rpm}$ for 15 minutes at $4^{\circ} \mathrm{C}$, and the plasma was separated and stored in a freezer at $-20^{\circ} \mathrm{C}$ until analysis. The calibration curves were prepared with Titrisol $^{\circledR}$ standard solution (Merck, Germany), at the following concentrations: $0.00,0.10,0.20,0.30,0.50$, and $1.00 \mu \mathrm{g} / \mathrm{mL}$. Aliquots of $600 \mu \mathrm{L}$ of plasma were diluted in deionized water at $1: 5$ ratio. After reading, the results were expressed as $\mu \mathrm{g} / \mathrm{dL}$. Analysis was conducted in duplicate, and the results were calculated from the average of the readings, establishing a coefficient of variation of less than $10 \%$. A range of $70-140 \mu \mathrm{g} / \mathrm{dL}$ was considered as the reference value for plasma copper for both sexes [29].

Analysis of serum ceruloplasmin and lipid profile was performed in a private certified laboratory. The nephelometry 
TABLE 1: Clinical, nutritional, and biochemical characteristics of the participants.

\begin{tabular}{|c|c|c|c|}
\hline Variable & Case group $(n=27)$ & Control group $(n=26)$ & $p$ value \\
\hline Age (years) ${ }^{\mathrm{a}}$ & $56.0(12.6)$ & $55.4(12.5)$ & 0.9989 \\
\hline \multicolumn{4}{|l|}{$\operatorname{Sex}^{c}$} \\
\hline Male & $13(48)$ & $11(42)$ & - \\
\hline Female & $14(52)$ & $15(58)$ & - \\
\hline BMI $(\mathrm{kg} / \mathrm{m})^{2 \mathrm{a}}$ & $22.6(2.9)$ & $27.3(4.7)$ & $<0.0001$ \\
\hline \multicolumn{4}{|l|}{ Initial manifestation $^{c}$} \\
\hline Spinal ALS & $20(74)$ & - & - \\
\hline Bulbar ALS & $7(26)$ & - & - \\
\hline Disease time in months ${ }^{\mathrm{b}}$ & $46(22-72)$ & - & - \\
\hline ALSFRS-R score ${ }^{\mathrm{b}}$ & $21(14-32)$ & - & - \\
\hline$>24$ points $^{\mathrm{c}}$ & $8(40)$ & - & - \\
\hline$\leq 24$ points $^{\mathrm{c}}$ & $12(60)$ & - & - \\
\hline \multicolumn{4}{|l|}{ Feeding pathway ${ }^{c}$} \\
\hline Oral & $21(78)$ & - & - \\
\hline Enteral & $5(18)$ & - & - \\
\hline Oral + enteral & $1(4)$ & - & - \\
\hline Dietary copper $(\mathrm{mg} / \mathrm{dia})^{\mathrm{b}}$ & $1.3(0.7-2.1)$ & $0.9(0.8-1.1)$ & 0.4122 \\
\hline Plasma copper $(\mu \mathrm{g} / \mathrm{dL})^{\mathrm{a}}$ & $133.9(26.5)$ & $164.1(25.7)$ & 0.0001 \\
\hline Serum ceruloplasmin $(\mathrm{mg} / \mathrm{dL})^{\mathrm{a}}$ & $23.2(6.3)$ & $25.0(4.2)$ & 0.2271 \\
\hline Total cholesterol $(\mathrm{mg} / \mathrm{dL})^{\mathrm{a}}$ & $190.6(45.6)$ & $190.2(41.9)$ & 0.9761 \\
\hline LDL-cholesterol $(\mathrm{mg} / \mathrm{dL})^{\mathrm{a}}$ & $121.3(38.1)$ & $124.1(35.9)$ & 0.7877 \\
\hline HDL-cholesterol (mg/dL) ${ }^{a}$ & $43.7(9.5)$ & $45.0(8.6)$ & 0.6162 \\
\hline Triglycerides $(\mathrm{mg} / \mathrm{dL})^{\mathrm{a}}$ & $126.0(55.4)$ & $124.9(70.9)$ & 0.9527 \\
\hline
\end{tabular}

${ }^{\mathrm{a}}$ Mean (standard deviation); ${ }^{\mathrm{b}}$ median (interquartile range); ${ }^{\mathrm{c}}$ frequency (percentage); BMI: body mass index; ALSFRS-R: ALS Functional Rating Scale-Revised.

method was used to measure serum ceruloplasmin and 21$53 \mathrm{mg} / \mathrm{dL}$ was considered as the reference range for both sexes. Serum total cholesterol (TC) and triglycerides (TGL) were measured by the enzymatic method and HDLcholesterol (HDL-c) by direct calorimetric method. The LDL-cholesterol values (LDL-c) were calculated using the Friedewald formula [30].

2.5. Statistical Analysis. The descriptive analysis was performed using measures of central tendency and dispersion, according to the data type. The Shapiro-Wilk test was applied to verify the normality of the data. To verify differences between the continuous variables, Student's $t$-test and Wilcoxon-Mann-Whitney test were used. The inferential analysis was performed by estimating the correlation between copper status and the lipid profile by Spearman correlation test.

The effect of independent variables on the outcome (discrete), measured by the ALSFRS-R score, was estimated after the univariate analysis. An adjusted model based on the Poisson distribution was used, as recommended for discrete outcomes in transversal studies. Dietary copper, plasma copper, serum ceruloplasmin, TC, LDL-c, HDL-c, and TGL were considered independent variables.

The cutoff point for the inclusion of the variables in the adjusted final model was $p<0.30$. In order to accept the final model, its significance was assessed using the chi-square test phrased as likelihood ratio. The variables were assessed separately by Wald chi-square test to estimate the regression coefficients. The presence of normality was tested for residues to guarantee the validity of the model. Statistical analysis was performed using
SPSS v.22, and a significance level of $5 \%$ was adopted for all analyses.

\section{Results}

Of the 53 subjects enrolled in the study, 27 comprised the case group and 26 the control group. The mean age of the participants in the case and control groups was 56 (12.6) and 55.4 (12.5) years, respectively. The case group presented a significantly lower BMI $(p<0.0001)$ compared with the control group. The case group had a disease time of 46 $(22-72)$ months, and $60 \%$ of the patients had $\leq 24$ points in the ALSFRS-R score (Table 1).

Dietary copper intake did not differ significantly between the groups $(p=0.4122)$. Also, the values of copper biomarkers and lipid profile were similar between the groups. However, plasma copper concentrations in the case group were significantly lower than those in the control group (133.9 versus $164.1 \mu \mathrm{g} / \mathrm{dL}, p=0.0001)$ (Table 1 ).

In the case group, plasma copper was positively correlated only with HDL-c $\left(r_{s}=0.398, p=0.044\right)$. In the control group, plasma copper was positively correlated not only with HDL-c $\left(r_{s}=0.479, p=0.015\right)$ but also with serum ceruloplasmin $\left(r_{\mathrm{s}}=0.646, p<0.001\right)$, TC $\left(r_{\mathrm{s}}=0.446, p=0.025\right)$, and LDL-c $\left(r_{\mathrm{s}}=0.445, p=0.029\right)$. Serum ceruloplasmin was positively correlated only with the LDL-c in the control group $\left(r_{\mathrm{s}}=0.407, p=0.043\right)$. Neither plasma copper nor serum ceruloplasmin was correlated with dietary copper (Table 2).

In the case group, dietary copper $(B=-0.373, p<0.001)$, plasma copper $(B=-0.005, p=0.033)$, and TC $(B=-0.312$, $p=0.001)$ were inversely associated with the ALSFRS-R score. Among them, dietary copper had the strongest 
TABLE 2: Correlation of plasma copper and serum ceruloplasmin with dietary copper and lipid profile of the participants.

\begin{tabular}{|c|c|c|c|c|c|c|}
\hline \multirow{2}{*}{ Variable } & \multicolumn{3}{|c|}{ Case group } & \multicolumn{3}{|c|}{ Control group } \\
\hline & $n$ & $r_{\mathrm{s}}$ & $p$ value & $n$ & $r_{\mathrm{s}}$ & $p$ value \\
\hline \multicolumn{7}{|l|}{ Plasma copper } \\
\hline Dietary copper & 27 & 0.194 & 0.333 & 23 & 0.054 & 0.807 \\
\hline Serum ceruloplasmin & 23 & 0.152 & 0.488 & 25 & 0.646 & $<0.001$ \\
\hline Total cholesterol & 27 & 0.293 & 0.138 & 25 & 0.446 & 0.025 \\
\hline LDL-cholesterol & 27 & 0.292 & 0.140 & 24 & 0.445 & 0.029 \\
\hline HDL-cholesterol & 26 & 0.398 & 0.044 & 25 & 0.479 & 0.015 \\
\hline Triglycerides & 27 & -0.208 & 0.297 & 25 & 0.371 & 0.068 \\
\hline \multicolumn{7}{|l|}{ Serum ceruloplasmin } \\
\hline Dietary copper & 23 & -0.145 & 0.508 & 24 & -0.184 & 0.390 \\
\hline Total cholesterol & 23 & 0.083 & 0.707 & 26 & 0.320 & 0.110 \\
\hline LDL-cholesterol & 23 & 0.053 & 0.810 & 25 & 0.407 & 0.043 \\
\hline HDL-cholesterol & 22 & 0.211 & 0.346 & 26 & 0.254 & 0.210 \\
\hline Triglycerides & 23 & -0.290 & 0.179 & 26 & 0.134 & 0.515 \\
\hline
\end{tabular}

$r_{s}:$ Spearman's correlation coefficient.

TABle 3: Association of Amyotrophic Lateral Sclerosis Functional Rating Scale-Revised (ALSFRS-R) with copper and lipid biomarkers in patients with amyotrophic lateral sclerosis.

\begin{tabular}{|c|c|c|c|c|c|c|c|}
\hline \multirow{2}{*}{ Variable } & \multirow{2}{*}{$B$} & \multirow{2}{*}{ Standard error } & \multicolumn{2}{|c|}{$95 \% \mathrm{CI}$} & \multicolumn{3}{|c|}{ Hypothesis testing } \\
\hline & & & Inferior & Superior & $x^{2}$ & $\mathrm{df}$ & $p$ value \\
\hline Model & & & & & 79.42 & 8 & $<0.001$ \\
\hline Interception & 3.779 & 0.4886 & 2.821 & 4.736 & 59.824 & 1 & $<0.001$ \\
\hline Dietary copper & -0.373 & 0.0784 & -0.527 & -0.219 & 22.646 & 1 & $<0.001$ \\
\hline Plasma copper & -0.005 & 0.0023 & -0.010 & 0.000 & 4.545 & 1 & 0.033 \\
\hline Serum ceruloplasmin & 0.016 & 0.0081 & 0.000 & 0.032 & 4.046 & 1 & 0.044 \\
\hline Total cholesterol & -0.312 & 0.0959 & -0.500 & -0.124 & 10.611 & 1 & 0.001 \\
\hline LDL-cholesterol & 0.314 & 0.0958 & 0.126 & 0.502 & 10.748 & 1 & 0.001 \\
\hline HDL-cholesterol & 0.308 & 0.0943 & 0.123 & 0.493 & 10.686 & 1 & 0.001 \\
\hline Triglycerides & 0.062 & 0.0190 & 0.025 & 0.099 & 10.568 & 1 & 0.001 \\
\hline
\end{tabular}

Dependent variable: ALSFRS-R score. $B$ : regression coefficient; $95 \%$ CI: $95 \%$ confidence interval; $\chi^{2}$ : chi-square test; df: degrees of freedom; $p$ value: probability of the null hypothesis is true.

association. On the other hand, serum ceruloplasmin $(B=0.016, p=0.044)$, LDL-c $(B=0.314, p=0.001)$, HDL-c $(B=0.308, p=0.001)$, and TGL $(B=0.062, p=0.001)$ were positively associated with the ALSFRS-R score (Table 3 ).

\section{Discussion}

The case group presented a significantly lower mean BMI compared with the control group. Weight loss and reduced BMI are associated with accelerated disease progression and lower survival rate [31]. Spinal-onset ALS, as found in most patients of this study, has less impairment in the swallowing ability than bulbar-onset ALS. This explains the use of enteral nutrition by the minority of the patients studied. The majority of patients presented low ALSFRS-R score, representing high disability and disease severity.

Our results showed that there was no difference in dietary copper intake between the groups. In addition, the median of dietary copper intake in the case and control groups was in agreement with the recommendations adopted [28]. Park et al. [32] found that the dietary copper intake among patients with ALS ranged from $0.9 \mathrm{mg} /$ day to $1.2 \mathrm{mg} /$ day. In our study, this variation was broader (Table 1), demonstrating a greater copper supply by the diet.
Contrary to our results, Forte et al. [33] found a significantly higher concentration of blood copper in patients with ALS $(103.5 \mu \mathrm{g} / \mathrm{dL})$ compared with the control group $(95.4 \mu \mathrm{g} / \mathrm{dL})$. However, other studies found no significant difference in plasma copper concentrations between ALS patients and healthy subjects [34, 35]. Differences in blood copper concentrations between studies may be explained by differences in food eating pattern, molecular profile of the population studied, and the method used to perform the lab tests. Considering the clinical heterogeneity among ALS patients, Forte et al. [33] suggest that the reference range for blood copper concentrations in subjects with and without ALS should be reviewed.

Mineral metabolism disturbances in patients with ALS may be due to several factors, such as malnutrition, malabsorption, increased excretion, and competition between minerals at binding sites [34]. Also, oxidative stress in patients with ALS may alter copper homeostasis $[6,36]$ and contribute to neurodegeneration [37]. At the same time, copper-deficient SOD-1 has a loss of antioxidant function and exerts a prooxidant function [38]. In addition, the level of copper deficiency seems to be proportional to the clinical severity of ALS [39]. 
In agreement with our results, some authors did not observe significant differences in the lipid profile between patients with ALS and healthy individuals $[8,40]$. Interestingly, some authors found significantly lower concentrations of TGL in male, but not in female, individuals with ALS compared with their control group [41]. Although there was no difference of serum lipids between males and females in both groups of our study (data not shown), Ikeda et al. [42] found significantly higher concentrations of TC, LDL-c, and TGL in female Japanese patients with ALS, compared with male ones. These controversial results may be explained by several genetic polymorphisms associated with lipid metabolism, able to develop individual biochemical phenotypes [43].

No correlation of dietary copper with plasma copper and serum ceruloplasmin was observed in both groups studied. In a recent review, Bost et al. [44] found that biomarkers of copper not necessarily respond to changes in dietary copper intake, probably due to homeostatic mechanisms controlling the absorption and excretion of this mineral. Also, there was no correlation between plasma copper and serum ceruloplasmin in the case group, but this correlation was positive in the control group. In healthy subjects, we expect to find a positive correlation between copper and ceruloplasmin, which is the main copper-binding protein [45]. Patients with ALS may have disturbances of ceruloplasmin activity [46] and lack of copper incorporation during ceruloplasmin biosynthesis [47], weakening a possible correlation between these two parameters.

In our study, the only correlation found between the copper biomarkers and the lipid profile in the case group was a positive correlation between plasma copper and HDL-c. It is possible that changes in serum lipids, oxidative stress, and alterations in copper metabolism in ALS are responsible for the absence of correlation between copper and serum lipids, differing from what was found in the control group. Several studies have found different associations between serum or plasma copper and serum lipids, demonstrating great metabolomic variation among individuals [14-19]. Circulating lipids are influenced by genetics, metabolism, habitual diet, lifestyle, and their interactions [48]. Moreover, disturbance of copper metabolism influences serum lipids through oxidative stress [49-51].

In our study, the association of ALSFRS-R with copper and lipid biomarkers was very divergent (Table 3). Dietary copper and plasma copper were inversely associated, while serum ceruloplasmin was positively associated with the functional status of ALS patients. Authors have found positive association between the concentration of copper in the nails of patients with ALS and functional status assessed by ALSFRS-R [52]. However, Peters et al. [34] found no association between the ALSFRS-R score and concentrations of plasma copper. Despite the conflicting results, it is known that copper is indirectly involved with motor neuron degeneration mechanisms in ALS by mutation of the copperdependent enzyme SOD-1, TDP-43 protein aggregation, and mitochondrial dysfunction [39]. Among the serum lipid biomarkers, only TC was inversely associated with the functional status of ALS patients studied. This fact may occur due to possible oxidation of cholesterol mediated by the copper as the disease progresses. On the other hand, the positive association found between the functional status and LDL-c, HDL-c, and TGL corroborates with the possible protective effect of hyperlipidemia in the survival of patients with ALS demonstrated in some studies [8-11].

The main limitations of this study were (1) the small number of participants in both groups due to the disease rarity and difficulty in finding healthy matches and (2) the few numbers of copper biomarkers evaluated. These limitations may have weakened the statistical power of the analysis and restricted deeper inferences in the discussion. Therefore, further studies are needed on this topic, considering larger samples and including other biomarkers of copper status and oxidative stress, as well as the presence of genetic polymorphisms related to lipid metabolism.

\section{Conclusion}

This study suggests a disturbance of copper status and its connection with the lipid profile in patients with ALS. Furthermore, copper status and lipid profile may influence the functional status of patients with ALS, standing out as potential biomarkers of disease severity.

\section{Data Availability}

Data are available upon request.

\section{Conflicts of Interest}

The authors declare that there are no conflicts of interest regarding the publication of this paper.

\section{Acknowledgments}

This work was supported in part by the Foundation for Research Support from the State of Rio Grande do Norte (FAPERN) and Coordination for the Improvement of Higher Education Personnel (CAPES), Grant no. 006/2014.

\section{References}

[1] D. Petrov, C. Mansfield, A. Moussy, and O. Hermine, "ALS clinical trials review: 20 years of failure. are we any closer to registering a new treatment?," Frontiers in Aging Neuroscience, vol. 9, no. 68, 2017.

[2] R. H. Brown and A. Al-Chalabi, "Amyotrophic lateral sclerosis," New England Journal of Medicine, vol. 377, no. 2, pp. 162-172, 2017.

[3] A. R. White, K. M. Kanninen, and P. J. Crouch, "Editorial: metals and neurodegeneration: restoring the balance," Frontiers in Aging Neuroscience, vol. 7, p. 127, 2015.

[4] G. Crisponi, V. M. Nurchi, D. Fanni, C. Gerosa, S. Nemolato, and G. Faa, "Copper-related diseases: from chemistry to molecular pathology," Coordination Chemistry Reviews, vol. 254, no. 7-8, pp. 876-889, 2010.

[5] F. Bozzo, A. Mirra, and M. T. Carrì, "Oxidative stress and mitochondrial damage in the pathogenesis of ALS: New perspectives," Neuroscience Letters, vol. 636, pp. 3-8, 2017.

[6] J. Y. Uriu-Adams and C. L. Keen, "Copper, oxidative stress, and human health," Molecular Aspects of Medicine, vol. 26, no. 4-5, pp. 268-298, 2005. 
[7] J. L. Burkhead and S. Lutsenko, "The role of copper as a modifier of lipid metabolism," in Lipid Metabolism, R. V. Baez, Ed., pp. 39-60, InTech, Rijeka, Croatia, 2013.

[8] R. Huang, X. Guo, X. Chen et al., "The serum lipid profiles of amyotrophic lateral sclerosis patients: a study from southwest China and a meta-analysis," Amyotrophic Lateral Sclerosis and Frontotemporal Degeneration, vol. 16, no. 5-6, pp. 359-365, 2015.

[9] N. A. Sutedja, Y. T. van der Schouw, K. Fischer et al., "Beneficial vascular risk profile is associated with amyotrophic lateral sclerosis," Journal of Neurology, Neurosurgery, and Psychiatry, vol. 82, no. 6, pp. 638-642, 2011.

[10] M. K. Rafiq, E. Lee, M. Bradburn, C. J. McDermott, and P. J. Shaw, "Effect of lipid profile on prognosis in the patients with amyotrophic lateral sclerosis: insights from the olesoxime clinical trial," Amyotrophic Lateral Sclerosis and Frontotemporal Degeneration, vol. 16, no. 7-8, pp. 478-484, 2015.

[11] J. Dorst, P. Kühnlein, C. Hendrich, J. Kassubek, A. D. Sperfeld, and A. C. Ludolph, "Patients with elevated triglyceride and cholesterol serum levels have a prolonged survival in amyotrophic lateral sclerosis," Journal of Neurology, vol. 258, no. 4, pp. 613-617, 2011.

[12] S. Lutsenko, "Human copper homeostasis: a network of interconnected pathways," Current Opinion in Chemical Biology, vol. 14, no. 2, pp. 211-217, 2010.

[13] D. Huster and S. Lutsenko, "Wilson disease: not just a copper disorder. Analysis of a Wilson disease model demonstrates the link between copper and lipid metabolism," Molecular BioSystems, vol. 3, no. 2, pp. 816-824, 2007.

[14] A. Tsuboi, M. Terazawa Watanabe, T. Kazumi et al., "Serum copper, zinc and risk factors for cardiovascular disease in community-living Japanese elderly women," Asia Pacific Journal of Clinical Nutrition, vol. 23, no. 2, pp. 239-245, 2014.

[15] N. M. Tasić, D. Tasić, P. N. Otašević et al., "Copper and zinc concentrations in atherosclerotic plaque and serum in relation to lipid metabolism in patients with carotid atherosclerosis," Vojnosanitetski Pregled, vol. 72, no. 9, pp. 801-806, 2015.

[16] K. N. Rai, N. S. Kumari, K. M. D. Gowda et al., "The evaluation of micronutrients and oxidative stress and their relationship with the lipid profile in healthy adults," Journal of Clinical and Diagnostic Research, vol. 7, no. 7, pp. 1314-1318, 2013.

[17] S. Bo, M. Durazzo, R. Gambino et al., "Associations of dietary and serum copper with inflammation, oxidative stress, and metabolic variables in adults," Journal of Nutrition, vol. 138, no. 2, pp. 305-310, 2008.

[18] S. C. V. C. Lima, R. F. Arrais, C. H. Sales et al., "Assessment of copper and lipid profile in obese children and adolescents," Biological Trace Element Research, vol. 114, no. 1-3, pp. 19-29, 2006.

[19] E. Al-khateeb, E. Al-zayadneh, O. Al-dalahmah et al., "relation between copper, lipid profile, and cognition in elderly jordanians," Journal of Alzheimer's Disease, vol. 41, no. 1, pp. 203-211, 2014.

[20] J.M. Cedarbaum, N. Stambler, E. Malta et al., "The ALSFRS-R: a revised ALS functional rating scale that incorporates assessments of respiratory function," Journal of the Neurological Sciences, vol. 169, no. 1-2, pp. 13-21, 1999.

[21] K. Guedes, C. Pereira, K. Pavan, B. Cataldo, and O. Valério, "Cross-cultural adaptation and validation of ALS functional rating scale-revised in Portuguese language," Arquivos de Neuro-Psiquiatria, vol. 68, no. 1, pp. 44-47, 2010.

[22] World Health Organization, "Physical status: the use and interpretation of anthropometry," Technical Report Series, World Health Organization, Geneva, Switzerland, 1995.
[23] W. C. Chumlea, A. F. Roche, and M. L. Steinbaugh, "Estimating stature from knee height for persons 60 to 90 years of age," Journal of the American Geriatrics Society, vol. 33, no. 2, pp. 116-120, 1985.

[24] W. C. Chumlea, S. S. Guo, and M. L. Steinbaugh, "Prediction of stature from knee height for black and white adults and children with application to mobility-impaired or handicapped persons," Journal of the American Dietetic Association, vol. 94, no. 12, pp. 1385-1388, 1994.

[25] F. E. Thompson and T. Byers, "Dietary assessment resource manual," Journal of Nutrition, vol. 124, no. 11, pp. 2245S2317S, 1994.

[26] S. M. Nusser, A. L. Carriquiry, K. W. Dodd, and W. A. Fuller, "A semiparametric transformation approach to estimating usual daily intake distributions," Journal of the American Statistical Association, vol. 91, no. 436, pp. 1440-1449, 1996.

[27] W. C. Willett, G. R. Howe, and L. H. Kushi, "Adjustment for total energy intake in epidemiologic studies," American Journal of Clinical Nutrition, vol. 65, no. 4, pp. 1220S-1228S, 1997.

[28] Institute of Medicine, Dietary Reference Intakes for Vitamin A, Vitamin K, Arsenic, Boron, Chromium, Copper, Iodine, Iron, Manganese, Molybdenum, Nickel, Silicon, Vanadium, and Zinc, The National Academies Press, Washington, DC, USA, 2001.

[29] D. S. Young, "Implementation of SI units for clinical laboratory data," Annals of Internal Medicine, vol. 106, no. 1, pp. 114-129, 1987.

[30] W. T. Friedewald, R. I. Levy, and D. S. Fredrickson, "Estimation of the concentration of low-density lipoprotein cholesterol in plasma, without use of the preparative ultracentrifuge," Clinical Chemistry, vol. 18, no. 6, pp. 499-502, 1972.

[31] S.T. Ngo, F. J. Steyn, and P. A. McCombe, "Body mass index and dietary intervention: implications for prognosis of amyotrophic lateral sclerosis," Journal of the Neurological Sciences, vol. 340, no. 1-2, pp. 5-12, 2014.

[32] Y. Park, J. Park, Y. Kim, H. Baek, and S. Hyun Kim, “Association between nutritional status and disease severity using the amyotrophic lateral sclerosis (ALS) functional rating scale in ALS patients," Nutrition, vol. 31, no. 11-12, pp. 1362-1367, 2015.

[33] G. Forte, B. Bocca, R. Oggiano et al., "Essential trace elements in amyotrophic lateral sclerosis (ALS): results in a population of a risk area of Italy," Neurological Sciences, vol. 38, no. 9, pp. 1609-1615, 2017.

[34] T. L. Peters, J. D. Beard, D. M. Umbach et al., "Blood levels of trace metals and amyotrophic lateral sclerosis," NeuroToxicology, vol. 54, pp. 119-126, 2016.

[35] P. M. Roos, O. Vesterberg, T. Syversen, T. P. Flaten, and M. Nordberg, "Metal concentrations in cerebrospinal fluid and blood plasma from patients with amyotrophic lateral sclerosis," Biological Trace Element Research, vol. 151, no. 2, pp. 159-170, 2013.

[36] M. Ikawa, H. Okazawa, T. Tsujikawa et al., "Increased oxidative stress is related to disease severity in the ALS motor cortex: a PET study," Neurology, vol. 84, no. 20, pp. 20332039, 2015.

[37] E. Tokuda, S. Watanabe, E. Okawa, and S. I. Ono, "Regulation of intracellular copper by induction of endogenous metallothioneins improves the disease course in a mouse model of amyotrophic lateral sclerosis," Neurotherapeutics, vol. 12, no. 2, pp. 461-476, 2015.

[38] A. Ahuja, K. Dev, R. S. Tanwar, K. K. Selwal, and P. K. Tyagi, "Copper mediated neurological disorder: visions into 
amyotrophic lateral sclerosis, Alzheimer and Menkes disease," Journal of Trace Elements in Medicine and Biology, vol. 29, pp. 11-23, 2015.

[39] F. J. Gil-Bea, G. Aldanondo, H. Lasa-Fernández, A. L. de Munain, and A. Vallejo-Illarramendi, "Insights into the mechanisms of copper dyshomeostasis in amyotrophic lateral sclerosis," Expert Reviews in Molecular Medicine, vol. 19, p. e7, 2017.

[40] A. Chiò, A. Calvo, A. Ilardi et al., "Lower serum lipid levels are related to respiratory impairment in patients with ALS," Neurology, vol. 73, no. 20, pp. 1681-1685, 2009.

[41] A. Wuolikainen, J. Acimovic, A. Lövgren-Sandblom, P. Parini, P. M. Andersen, and I. Björkhem, "Cholesterol, oxysterol, triglyceride, and coenzyme Q homeostasis in ALS. Evidence against the hypothesis that elevated 27-hydroxycholesterol is a pathogenic factor," PLoS One, vol. 9, no. 11, Article ID e113619, 2014.

[42] K. Ikeda, T. Hirayama, T. Takazawa, K. Kawabe, and Y. Iwasaki, "Relationships between disease progression and serum levels of lipid, urate, creatinine and ferritin in Japanese patients with amyotrophic lateral sclerosis: a cross-sectional study," Internal Medicine, vol. 51, no. 12, pp. 1501-1508, 2012.

[43] M. L. Curti, P. Jacob, M. C. Borges, M. M. Rogero, and S. R. G. Ferreira, "Studies of gene variants related to inflammation, oxidative stress, dyslipidemia, and obesity: implications for a nutrigenetic approach," Journal of Obesity, vol. 2011, Article ID 497401, 31 pages, 2011.

[44] M. Bost, S. Houdart, M. Oberli, E. Kalonji, J. F. Huneau, and I. Margaritis, "Dietary copper and human health: current evidence and unresolved issues," Journal of Trace Elements in Medicine and Biology, vol. 35, pp. 107-115, 2016.

[45] J. Osredkar and S. Natasa, "Copper and zinc, biological role and significance of copper/zinc imbalance," Journal of Clinical Toxicology, vol. S3, pp. 1-18, 2011.

[46] G. Tórsdóttir, J. Kristinsson, G. Gudmundsson, J. Snaedal, and T. Johannesson, "Copper, ceruloplasmin and superoxide dismutase (SOD) in amyotrophic lateral sclerosis," Pharmacology and Toxicology, vol. 87, no. 3, pp. 126-130, 2000.

[47] E. Sedlak and P. Wittung-Stafshede, "Discrete roles of copper ions in chemical unfolding of human ceruloplasmin," Biochemistry, vol. 46, no. 33, pp. 9638-9644, 2007.

[48] Y. Zheng and L. Qi, "Diet and lifestyle interventions on lipids: combination with genomics and metabolomics," Clinical Lipidology, vol. 9, no. 4, pp. 417-427, 2014.

[49] R. Gutiérrez-García, T. Pozo, M. Suazo, V. Cambiazo, and M. González, "Physiological copper exposure in Jurkat cells induces changes in the expression of genes encoding cholesterol biosynthesis proteins," BioMetals, vol. 26, no. 6, pp. 1033-1040, 2013.

[50] Y. Liu, H. Yang, Z. Song, and S. Gu, "Copper excess in liver HepG2 cells interferes with apoptosis and lipid metabolic signaling at the protein level," Turkish Journal of Gastroenterology, vol. 25, no. S1, pp. 116-121, 2014.

[51] A. Morrell, S. Tallino, L. Yu, and J. L. Burkhead, "The role of insufficient copper in lipid synthesis and fatty-liver disease," IUBMB Life, vol. 69, no. 4, pp. 263-270, 2017.

[52] M. Bergomi, M. Vinceti, G. Nacci et al., "Environmental exposure to trace elements and risk of amyotrophic lateral sclerosis: a population-based case-control study," Environmental Research, vol. 89, no. 2, pp. 116-123, 2002. 


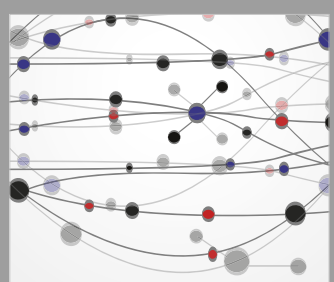

The Scientific World Journal
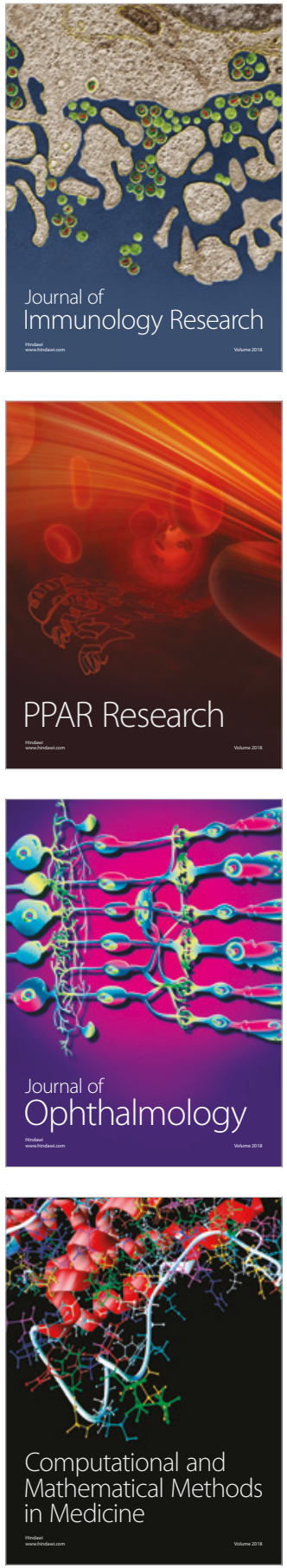

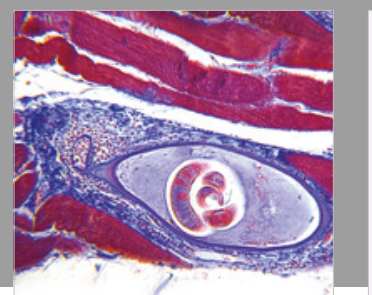

Gastroenterology Research and Practice

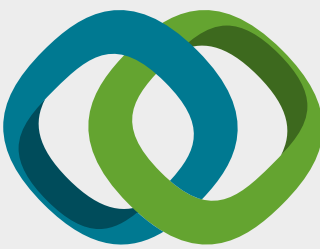

\section{Hindawi}

Submit your manuscripts at

www.hindawi.com
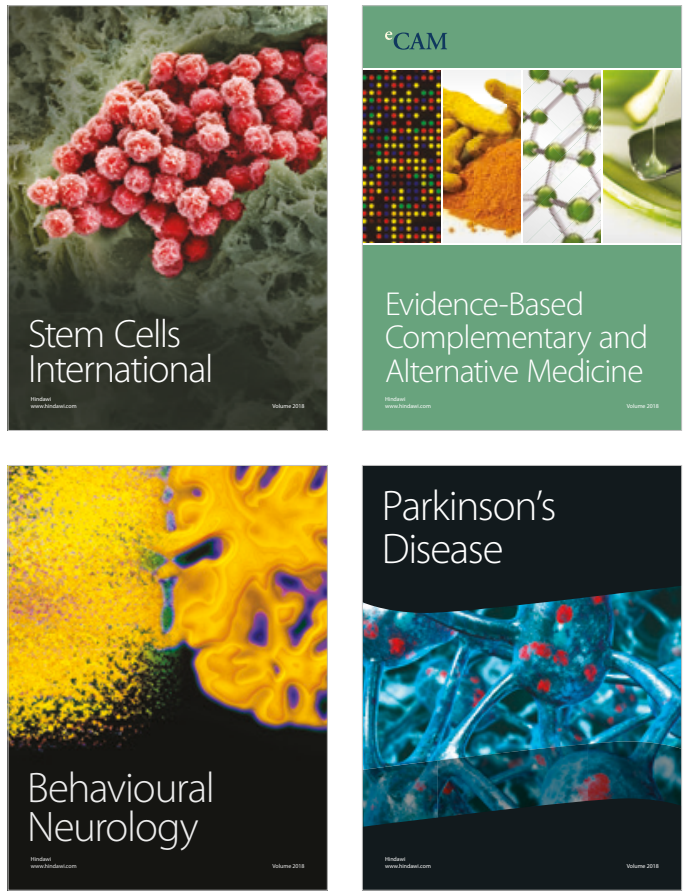

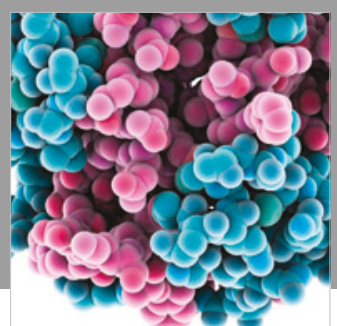

ournal of

Diabetes Research

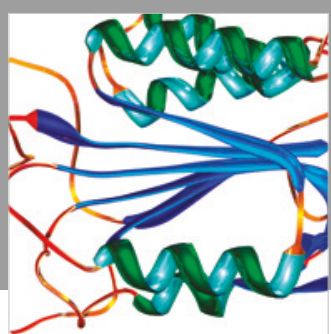

Disease Markers
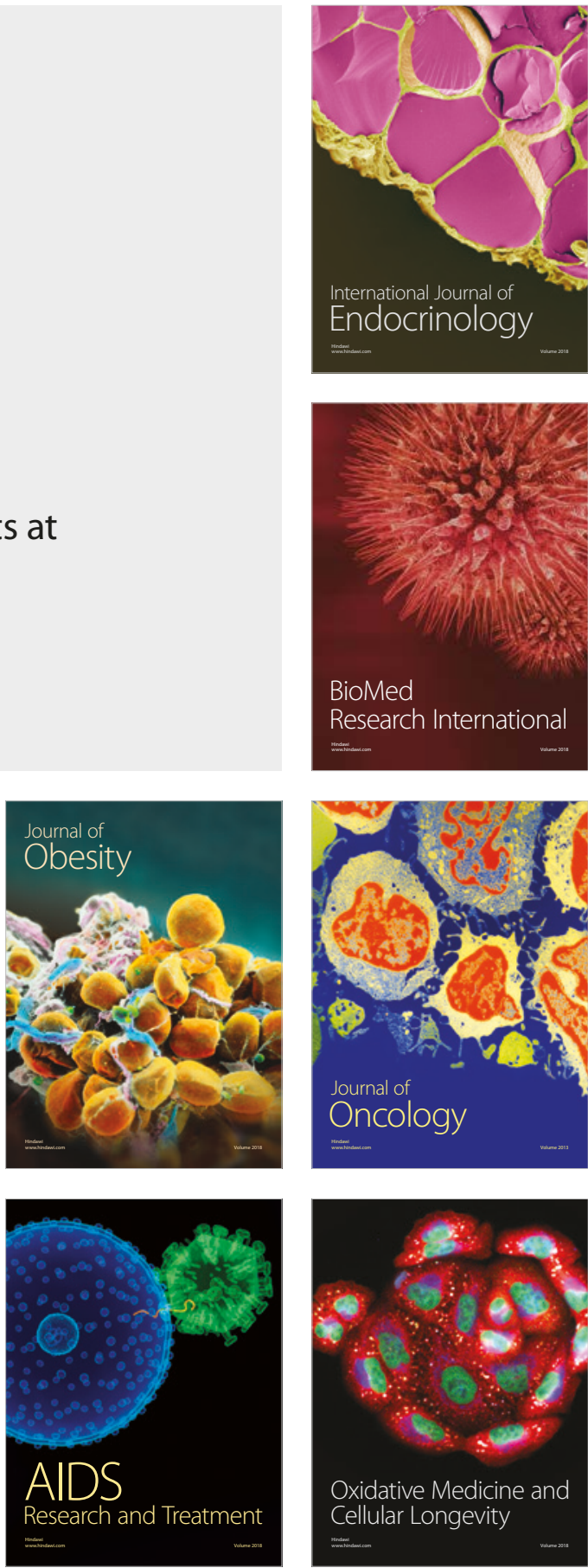